\title{
Schmetterlingswiese, Bienenschmaus und Hummelmagnet - Insektenrettung aus der Samentüte?
}

Corinne Buch \& Armin Jagel

\begin{abstract}
In order to mitigate the decline of insects, many retailers offer seed mixtures that can be dispersed in the yard or anywhere outside. In many cases, conservation groups support this practice. However, the content in those mixtures is often not documented and, in many cases, includes of non-native and annual species with no evidence of a long-lasting positive effect. If a positive effect is observed, it mostly benefits already the common insects and not those insect species and not thoses in decline. The complexity of insect decline and seed mixtures as well as alternatives to the undirected seed dispersal movement are outlined.
\end{abstract}

\section{Zusammenfassung}

Als Maßnahme gegen das Insektensterben werden Samentütchen im Handel angeboten oder von Firmen und Behörden verteilt, die im Garten, aber auch in der freien Landschaft, ausgestreut werden. Häufig wird dies von Naturschutzverbänden unterstützt. Der Inhalt dieser Tütchen ist in der Regel nicht dokumentiert und besteht in vielen Fällen aus nicht-einheimischen, einjährigen Arten, die zu keinem nachhaltigen, positiven Effekt in der Natur führen und höchstens den häufigen Insektenarten nützen. Es werden die Komplexität der Themenfelder Insektensterben und Ansaaten beleuchtet und Handlungsalternativen zur ungezielten Samenaussaat aufgezeigt.

\section{Einleitung}

Das Insektensterben geht gerade durch alle Medien. Doch was genau ist damit gemeint? Trotz einiger Probleme, die die Imkerei betreffen, geht es dabei nicht um eine Gefährdung der domestizierten Honigbiene, die einen hohen und konstanten Schutz erfährt. Es betrifft vielmehr ein komplexes Themenfeld um die heimischen, wildlebenden Insekten, das selbst mit fundiertem Fachwissen kaum $\mathrm{zu}$ überblicken ist und deshalb in vielen Medien stark vereinfacht und dadurch verzerrt dargestellt wird. Im Wesentlichen umfasst das Thema drei relativ unterschiedliche Aspekte: Den Rückgang der undifferenzierten Biomasse an Fluginsekten (s. Untersuchungen bei Hallmann et al. 2017), die immer länger werdenden Roten Listen, welche Arten und Artengruppen differenziert betrachten (z. B. Binot-Hafke et al. 2011) sowie den allgemeinen Verlust an Biodiversität, womit nicht nur die Artenvielfalt, sondern laut Definition auch die genetische Vielfalt sowie die Vielfalt an Biotoptypen in einer Landschaft gemeint sind (WITTIG \& NieKISCH 2014).

Die Gründe für das Insektensterben sind so komplex wie das Thema selbst, aber ganz offensichtlich lässt sich vieles auf die industrialisierte Landwirtschaft zurückführen. Sei es die Arten- armut im Intensivgrünland und auf Äckern, die ausgeräumte, strukturarme Agrarlandschaft ohne Säume, oder die Überdüngung und der Pestizideinsatz. Dadurch sind mittlerweile vielerorts die Städte zu Zentren der Artenvielfalt geworden (Brosch et al. 2014, Buch 2018), während die sogenannte Naturlandschaft immer stärker verarmt. Aber auch im Siedlungsbereich herrscht ein ständiger Konflikt zwischen dem Erhalt artenreicher Freiflächen und Versiegelung und Flächenverbrauch. Auch das große Potential der Straßenränder, die in der Summe eine beachtliche Fläche darstellen, bleibt für die Artenvielfalt weitgehend ungenutzt, wenn die dortigen Grünflächen zu häufig und zum falschen Zeitpunkt gemäht bzw. gemulcht statt gemäht werden.

Allerdings spielt die aktuelle Medienpräsenz des Insektensterbens dem Naturschutz in gewisser Weise auch in die Karten: Es sind dadurch grundsätzliche Sachverhalte bereits im Bewusstsein der Bevölkerung verankert und es herrscht eine hohe Bereitschaft, aktiv zu werden. Doch während es offensichtlich seitens des ehrenamtlichen und behördlichen Naturschutzes oft noch an Orientierung, einheitlichem Rat und Kommunikationsstrategien mangelt, haben Marktwirtschaft und Politik die Zeichen der Zeit rasch erkannt: In 


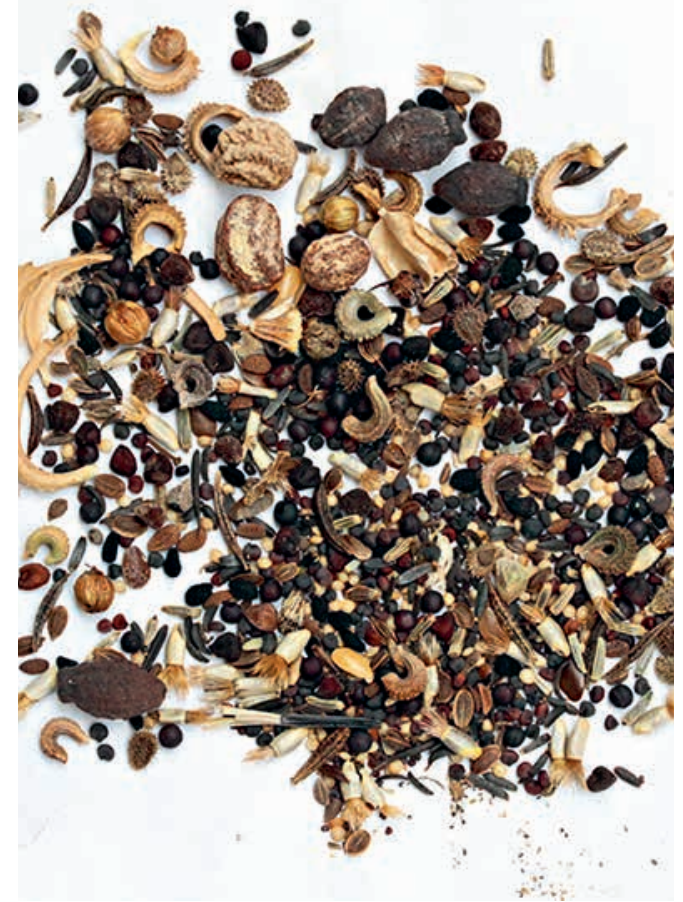

Abb. 1: Inhalt einer Samenmischung für eine Schmetterlingswiese aus einem Baumarkt (Mülheim, 28.04.2018). (Foto: C. BuCH)

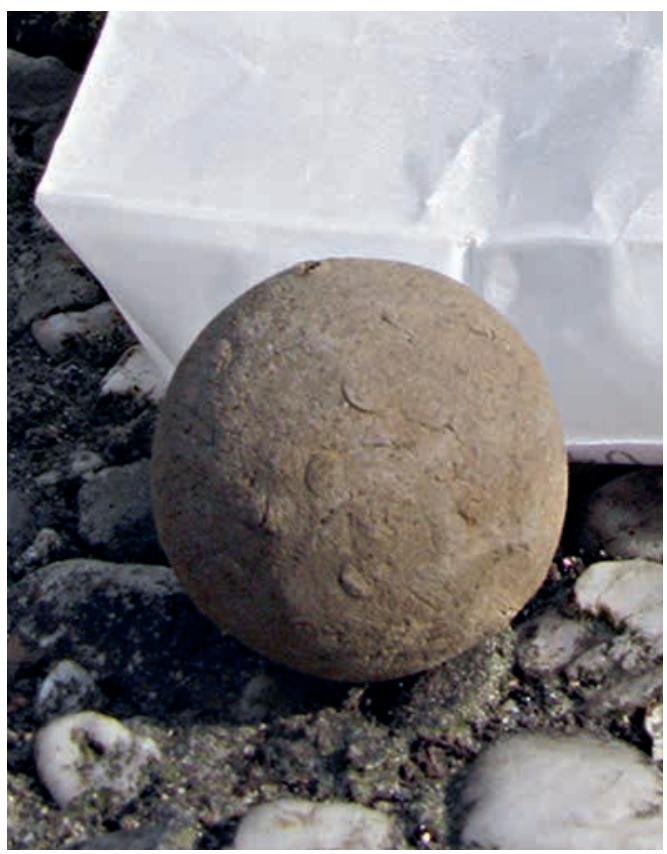

Abb. 2: Seedbomb im Rahmen des Guerilla Gardenings für eine buntere Stadt (Bochum, 20.05.2011). (Foto: A. JAGEL) so gut wie jedem Baumarkt, Discounter, aber auch auf Werbeveranstaltungen sind mittlerweile Samentütchen für „die Bienen“ erhältlich, die sich entweder für verhältnismäßig teures Geld oder aber als Maßnahme für einen ökologischen Anstrich von Firmen und Parteien gut unter die Leute bringen lassen. Ansprechende Namen wie Schmetterlingswiese, Bienenschmaus und Hummelmagnet auf einem attraktiven Cover mit bunten Blumen halten jedoch ihr ökologisches Versprechen nur selten.

\section{Samentüten und ihr Inhalt}

Zur genaueren Analyse des Sachverhalts lohnt sich zunächst ein Blick auf Vorder- und Rückseite der Samentütchen. Häufig findet man lediglich Beschreibungen wie Sommerblumenmischung, Wildblumen oder aber phantasievolle Bezeichnungen wie Blaues Wunder ohne jegliche Angabe über die enthaltenen Arten. Spätestens an dieser Stelle sollten erste Zweifel an einer ökologisch tauglichen Zusammensetzung aufkommen. Hinzu kommen häufig auf die Packung aufgedruckte Gütesiegel, die den angeblich besonderen Wert des Produktes kennzeichnen, aber vom Anbieter frei erfunden und daher ohne jegliche ökologische Relevanz sind. Die Abbildungen der Mischungen auf dem Cover sind oft nur beispielhaft ausgewählt und geben nicht unbedingt den realen Tüteninhalt wieder, daher lag es nahe, einmal durch Aussaatversuche herauszufinden, was diese Tütchen wirklich enthalten (Abb. 1).

Hierzu wurden die Samen fotografiert und ausgesät. Zusätzlich wurden auch vergleichbare Produkte wie Seedbombs kontrolliert ausgesät (Abb. 2). Auch die Keimlinge und die daraus wachsenden Pflanzen wurden fotografisch dokumentiert, bis sie letztendlich bestimmt werden konnten.

Das Ergebnis der Ansaaten war ernüchternd: In fast jedem Samentütchen, auch in denen mit der Aufschrift Wildblumen, was dem Kunden ganz offensichtlich suggerieren soll, dass es sich um heimische Arten handelt, waren ausschließlich oder fast ausschließlich nicht-einheimische Arten, die man auch getrennt als Samentütchen 


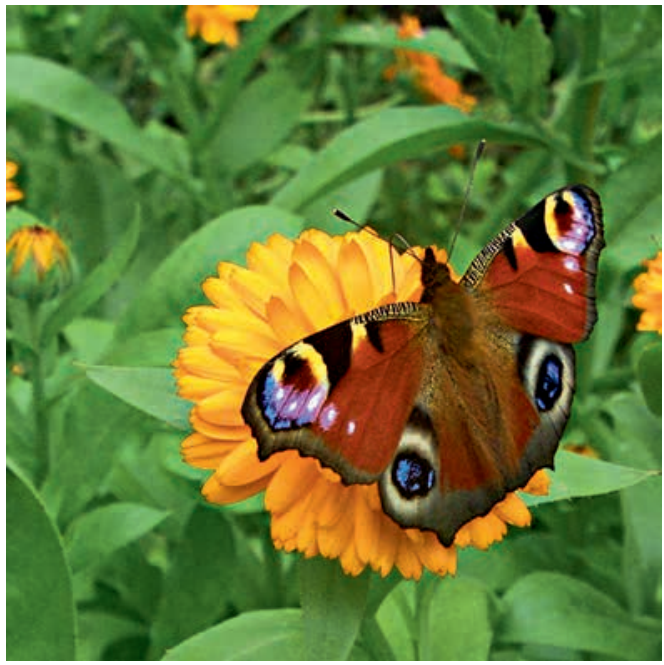

Abb. 3: Tagpfauenauge (Aglais io) auf einer Ringelblume. (Foto: A. JAGEL)

im Repertoire der Sommerblumen erwerben kann. Die in den Mischungen bemerkenswerterweise fast durchweg einjährigen Arten stammten aus dem Mittelmeergebiet, aus Südosteuropa oder auch aus ganz anderen Florenregionen wie Nord- oder Mittelamerika (Abb. 3-9). Eine der wenigen heimischen Arten, die fast in jeder Bienenmischung auftritt, ist Centaurea cyanus (Kornblume), jedoch in verschiedenen Farbvarianten von Weiß über Rosa bis zum gewohnten Blau. Weitere

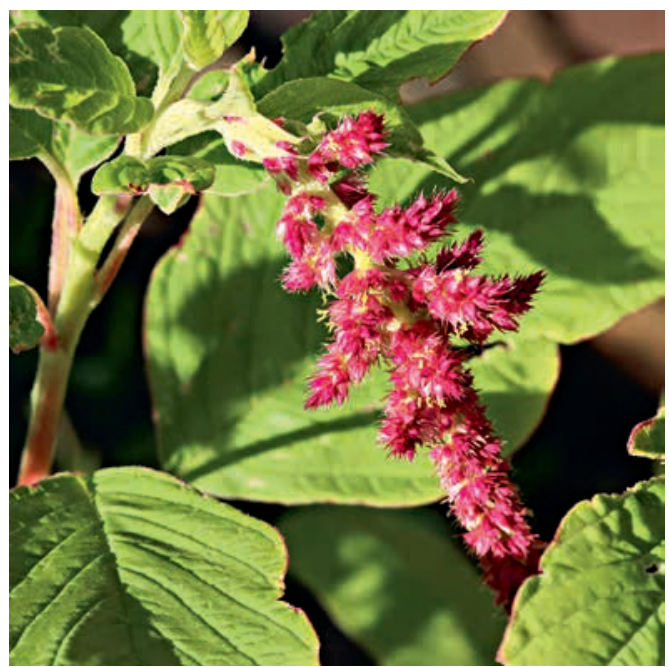

Abb. 5: Amaranthus caudatus (Garten-Fuchsschwanz, (Bochum, 30.07.2018). (Foto: A. JAGEL)

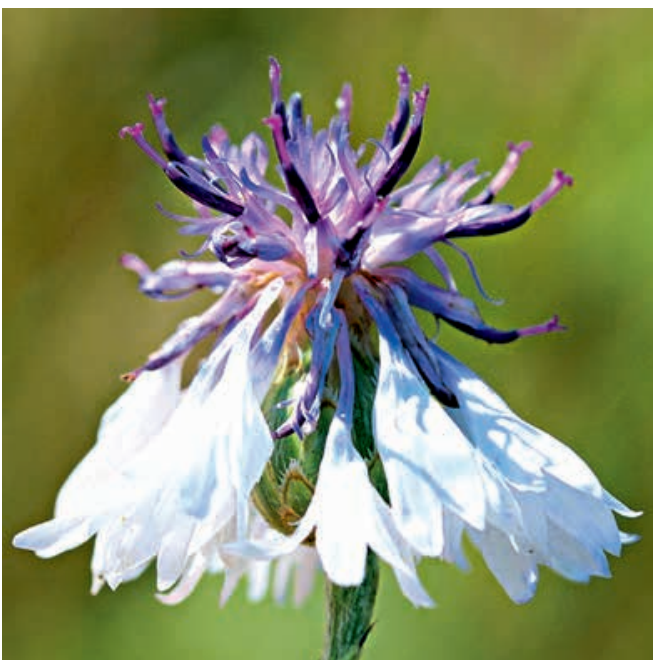

Abb. 4: Centaurea cyanus (Kornblume) mit weißen Randblüten (Mülheim/Ruhr, 18.08.2018). (Foto: C. BucH)

regelmäßige Vertreter sind Agrostemma gracile (Zierliche Kornrade, Türkei, Israel), Amaranthus caudatus (Garten-Fuchsschwanz, Südamerika), Ammi majus (Große Knorpelmöhre, Mittelmeergebiet), Calendula officinalis (Garten-Ringelblume, Herkunft unbekannt), Convolvulus tricolor (Dreifarbige Winde, Mittelmeergebiet), Coreopsis tinctoria (Färber-Mädchenauge, Nord-Amerika), Cosmos bipinnatus (Schmuckkörbchen, Nord- \& Mittel-Amerika), Dianthus barbatus (Bart-Nel-

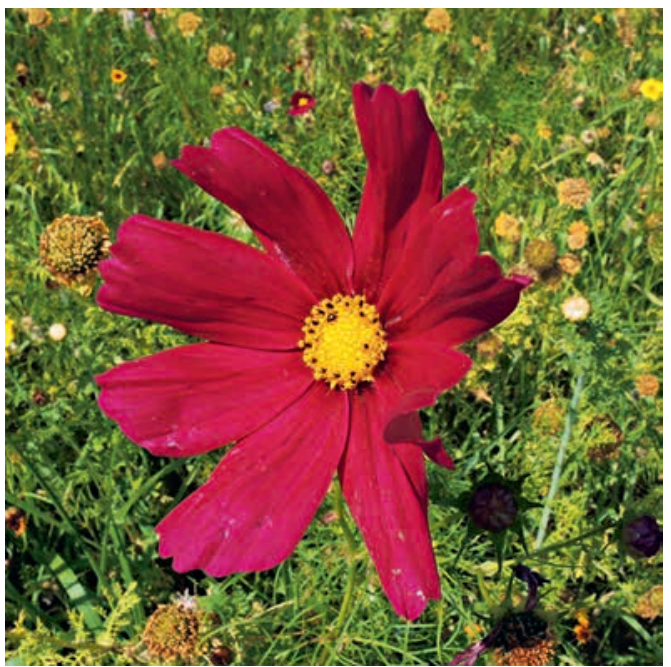

Abb. 6: Cosmos bipinnatus (Schmuckkörbchen, Bochum, 18.07.2018). (Foto: A. JAGEL) 
ke, Ost-Europa, Asien), Eschscholzia californica (Kalifornischer Kappenmohn, Nord-Amerika), Glebionis coronaria (Kronen-Wucherblume, Mittelmeergebiet), Lavatera trimestris (Bechermalve, Mittelmeergebiet), Linaria maroccana (Marokko-Leinkraut, Marokko), Linum grandiflorum (Roter Lein, Algerien), Lobularia maritima (Strand-Silberkraut, Mittelmeergebiet), Lychnis coronaria (Kronen-Lichtnelke, Südost-Europa, Kleinasien) u. v. m.

Vermutlich wurden vor allem Arten ausgewählt, die sich effektiv vermehren und deren Samen sich entsprechend kostengünstig herstellen lassen. Möglicherweise stammt ein nicht unerheblicher

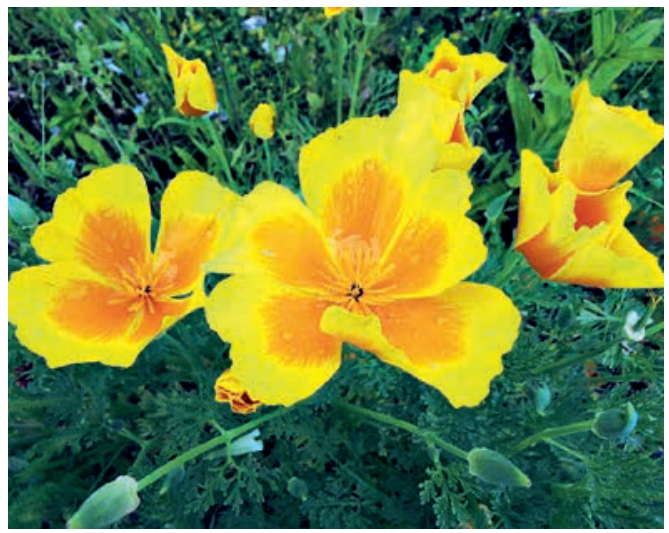

Abb. 7: Eschscholzia californica (Kalifornischer Kappenmohn, Bielefeld, 29.05.2016). (Foto: A. JAGEL)

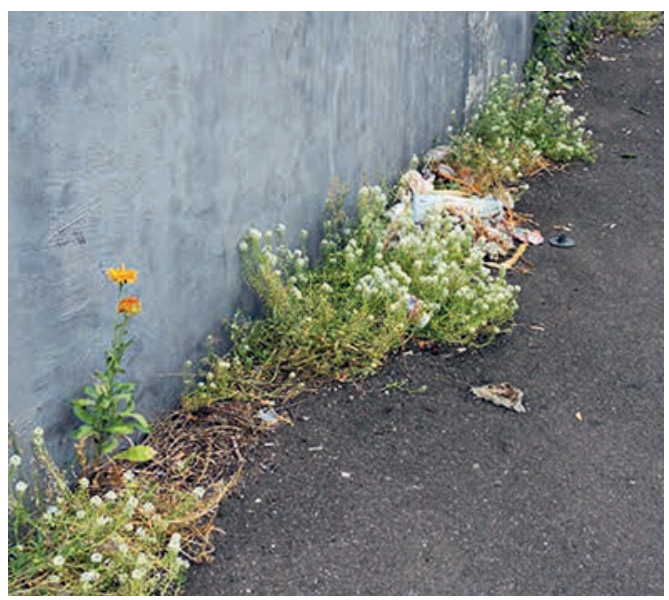

Abb. 8: Lobularia maritima (Strand-Silberkraut) und Calendula officinalis (Garten-Ringelblume) auf einem Bürgersteig in Köln-Worringen (18.09.2016). (Foto: A. JAGEL)
Teil in den Samenmischungen sogar aus Überschüssen sonstiger Produktionen, die sich in den undefinierten Mischungen noch gut vermarkten lassen.

Wenn die Arten nicht auf dem Tütchen aufgelistet sind, kann der Inhalt ohne Konsequenzen variieren. Aber auch auf die Aussaat von Samen aus definiertem Saatgut mit nur einer Art ist nicht unbedingt Verlass. So enthielt eine Samentüte nicht das auf dem Cover angegebene, in Deutschland seltene und stark gefährdete Ackerunkraut Sommer-Adonisröschen (Adonis aestivalis, vgl. BfN 2018), sondern das im Mittelmeergebiet heimische Herbst-Adonisröschen (Adonis annua), das in Deutschland als unbeständiger Neophyt auftritt (Floraweb 2018). Ähnliches passierte auch gattungsübergreifend bei Ipomoea purpurea (Purpur-Prunkwinde) und Convolvulus tricolor (Dreifarbige Winde), verkauft als Ipomoea tricolor. Beide Gattungen lassen sich aber schon aufgrund der Keimblätter eindeutig unterscheiden.

\section{Konsequenzen}

\subsection{Funde typischer Pflanzen aus Samentüten in der Landschaft: Bestimmung und Status}

Seit einiger Zeit wird von Botanikern eine auffällige Häufung von Funden neuer Arten in der Landschaft gemeldet. Zunächst fielen diese als exotische Arten auf, die mit gängiger Literatur oft gar nicht bestimmt werden konnten. Die Ansprache erforderte also eine mehr oder weniger aufwändige Recherche, teils auch den Blick in Bestimmungsbücher der Mittelmeerflora. Es wurde schnell deutlich, dass diese Funde in Zusammenhang mit dem Vertrieb der einschlägigen Samentütchen und dem Insekten-Hype stehen. Die Vorkommen dieser Arten im Gelände stammen entweder direkt von Menschen, die gut gemeint die Landschaft zum Schutz der Insekten ökologisch aufwerten wollen, oder aber sie treten bereits aus solchen Ansaaten verwildernd auf.

Ein deutliches Zeichen für gezielte, direkte Ansaaten in der Landschaft ist das Vorkommen von gleich mehreren typischen Bienentüten-Arten 


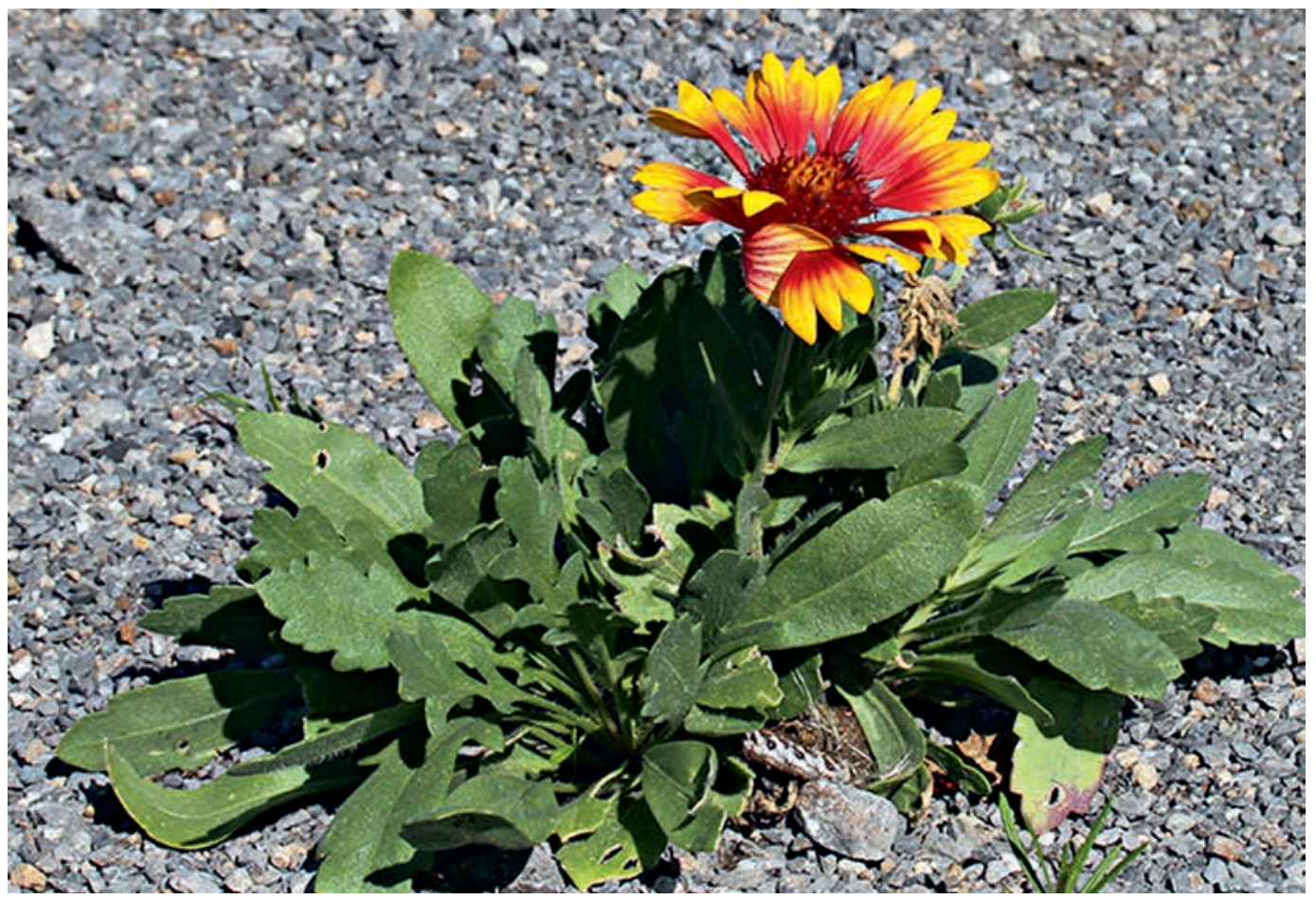

Abb. 9: Gaillardia aristata (Prärie-Kokardenblume) auf der Halde Rheinelbe in Gelsenkirchen (21.07.2017). (Foto: C. BuCH)

an einem Ort. In solchen Fällen kann davon ausgegangen werden, dass der Standort Opfer einer Guerilla Gardening-Aktion wurde. In vielen Fällen bleibt dies jedoch völlig unklar, z. B. wenn nur einzelne Arten bzw. Pflanzen auftreten oder diese an schlecht zugänglichen Orten wachsen.

Letztlich erschwert dies insgesamt die Beurteilung, ob eine Art einfach nur häufig in Mischungen enthalten ist und deshalb zunehmend in der Landschaft auftritt, oder ob sie, bedingt durch Ansalbungen, dabei ist, sich zu etablieren. Eine Klärung ist jedoch sowohl wissenschaftlich als auch aus Sicht des Naturschutzes bedeutsam. Im schlimmsten denkbaren Szenario könnte sich eine Art als invasiv herausstellen und heimische Arten, zumindest am jeweiligen Wuchsort, verdrängen. Dies ist zunächst äußerst unwahrscheinlich, da der Großteil der Arten nicht winterhart ist und daher im folgenden Jahr nicht wieder aufkommt. Allerdings steigt die Wahrscheinlichkeit mit der Masse an ausgestreuten Samen aus den Samentütchen und mit der steigenden Vielfalt von zu diesem
Zweck im Handel verfügbaren Arten (DeHnenSchmutz et al. 2007).

\subsection{Aktionismus außer Kontrolle}

Obwohl die Idee von Guerilla Gardening schon seit Jahrzehnten existiert, erlebt die Bewegung durch die aktuelle öffentliche Diskussion um das Insektensterben einen enormen Aufschwung und ist mittlerweile nicht nur gesellschaftlich völlig akzeptiert, sondern wird auch für kommerzielle Zwecke genutzt, was genau genommen der Grundidee des Guerilla Gardenings widerspricht.

Durch den aktuellen Trend wird die Landschaft nun jedoch völlig willkürlich angereichert. Vom Einzelkämpfer bis zur Kindergruppe von Naturschutzverbänden werden unkontrolliert Seed Bombs geworfen oder Guerilla Gardening-Aktionen durchgeführt, mit den besten Absichten, jedoch ohne ausreichende Arten- und Ortskenntnisse. Sei es die Baumscheibe vor der Haustür, der zu sterile Schottergarten des Nachbarn, die Industriebrache oder auch der Waldsaum und die 


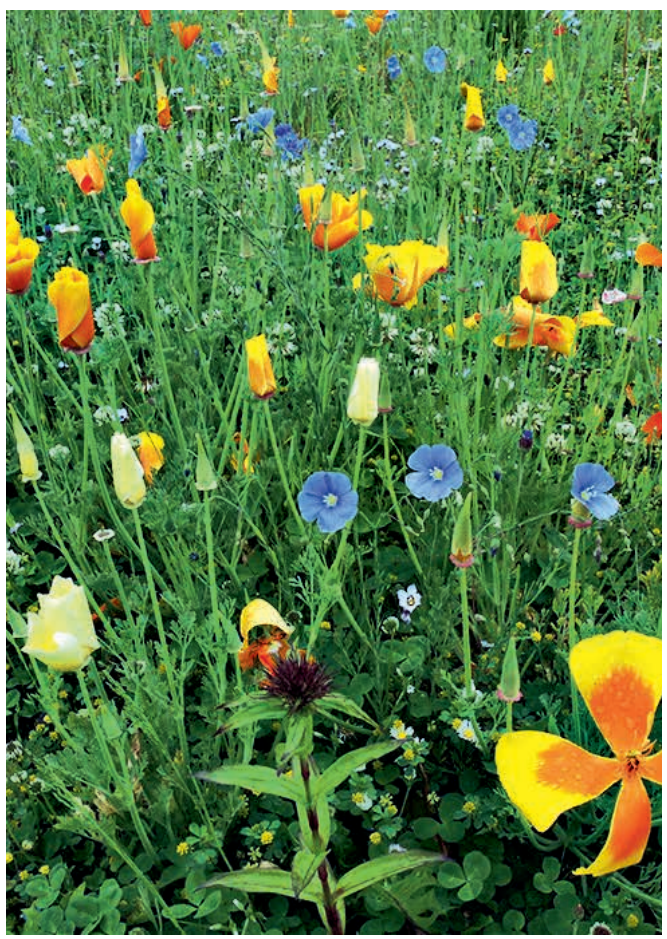

Abb. 10: „Einjährige Wiese“ aus der Samentüte mit verschiedenfarbigem Kalifornischem Kappenmohn, Bart-Nelke und Lein, Bielefeld, 05.06.2016). (Foto: A. JAGEL)

Bachaue im Naturschutzgebiet. Alles wird wild begärtnert - im festen Glauben, etwas Gutes für die Natur und für die Bienen zu tun. Was jedoch an der Baumscheibe oder in Nachbars Garten lediglich eine Verschwendung von Zeit, Ressourcen und Geld ist, dabei aber weitgehend ohne Konsequenzen bleibt, da die Arten dort schnell wieder verschwinden, kann sich an naturnahen Standorten möglicherweise negativ auswirken. Gerade im städtischen Raum existiert eine Reihe von Lebensräumen, die bereits wertvoll und artenreich sind, allerdings für den Laien eher karg und leblos anmuten. Das beste Beispiel sind die floristisch und faunistisch gut untersuchten Brachen und Ruderalflächen mit ihren zahllosen Arten der Roten Listen, die hier einen Ersatzstandort für ihre zerstörten Lebensräume in der Kulturlandschaft finden (z. B. Jagel \& Gausmann 2010, Buch \& KeIL 2013). Es ist aktuell noch völlig unklar, wie sich Ansaaten auf solche Lebensräume auswirken. Klar scheint jedoch: Die wünschenswerteste Variante dürfte die sein, dass sie keinen
Effekt haben und auf dem Extremstandort nicht lange überdauern.

\section{Täuschungen und Missverständnisse}

\subsection{Einjährige Wiese?}

Ein wesentlicher Punkt, der nicht nur dem Vegetationskundler direkt auffällt, ist der häufig verwendete Begriff der einjährigen Wiese. Wiesen sind immer Pflanzengesellschaften, die durch mehrjährige Pflanzenarten charakterisiert sind. Durch das Mähen werden die oberirdischen Pflanzenteile einige Zentimeter über dem Boden rigoros entfernt, es setzen sich also diejenigen Pflanzen durch, deren Erneuerungsorgane unterhalb des Mähers liegen. Einjährige Arten kommen zwar in Wiesen auch vor, sind aber nicht bestandsbildend und meist mehr oder weniger regelmäßige Begleiter. Ebenso irreführend sind Begriffe wie Bienenweide, da eine Weide über den Fraß der Gräser und Beikräuter sowie den Tritt durch Nutztiere definiert ist (Dierschke \& Briemle 2002).

Eine Saatmischung mit einjährigen Arten, die zudem noch im Hochsommer blühen, ist für die Nutzung als Mähwiese somit eine denkbar ungeeignete Wahl (Abb. 10, 11). Aber auch ohne Mahd wachsen einjährige Pflanzenarten, sofern sie nicht aus mitteleuropäischem Klima stammen, im nächsten Jahr kaum mehr nach. Es muss also nicht nur eine neue Tüte gekauft werden, sondern für eine erneute Einsaat auch der Boden umgebrochen werden - der Tod für alle Insekten, deren Larven in abgestorbenen Halmen oder in Bodennähe überwintern.

\subsection{Effekt für die Insektenwelt}

Es stellt sich die Frage nach dem Effekt dieser Mischungen auf die Insektenwelt. Ist eine Samentüte nicht allemal besser als ein totgespritzter Ackerrand oder ein steriler Schottergarten?

Dies kann kaum pauschal beantwortet werden, da gerade faunistische Gefüge sehr komplex sind. Festzustellen ist allerdings, dass gerade in den wenigen Wochen des Hauptblühaspekts zwar reichlich Insekten an den Blüten solcher Aussaaten zu finden sind, es sich jedoch in der Regel um 
häufige Arten ohne besondere Ansprüche handelt wie z. B. Tagpfauenauge, Kleiner Kohlweißling und die allgegenwärtige Honigbiene. Hier wäre also lediglich ein positiver Effekt auf die absolute Biomasse an Insekten nachvollziehbar. Betrachtet man jedoch die Arten der Roten Listen, wird klar, dass es sich bei den stark gefährdeten und vom Aussterben bedrohten Insektenarten mehrheitlich um auf bestimmte heimische Pflanzenarten stark spezialisierte Arten handelt, die auf intakte Artengemeinschaften, natürliche Lebensräume und traditionelle Bewirtschaftung angewiesen sind.

\subsection{Schutz von Honigbienen?}

Eines von vielen Missverständnissen ist die im Sprachgebrauch mangelnde Differenzierung zwischen Honigbienen und Wildbienen. „Die Bienen" werden von vielen Laien und auch $\mathrm{z}$. T. von Presse und Rundfunk als Honigbiene verstanden. Dies beschert den Imkern momentan großen $\mathrm{Zu}$ spruch, da für die Honigbiene getrommelt wird. Projekte zum Aufstellen von Bienenstöcken werden ins Leben gerufen und von der Presse ganzjährig begleitet. Auch die schon seit Längerem und oft gerühmten sogenannten Blühstreifen am Ackerrand kommen wieder ins Gespräch, obwohl auch diese immer schon eng auf die Bedürfnisse der Honigbienen zugeschnitten waren und daher zahlreiche nicht einheimische Arten enthalten, die von den meisten gefährdeten Insektenarten nicht genutzt werden können. Außerdem belegen zahlreiche Untersuchungen, dass die Honigbiene für Wildbienen eine Konkurrenz darstellt und mit ihnen um die knapp gewordenen Nahrungsressourcen konkurriert, aber auch weitere negative Effekte mit sich bringen kann, z. B. Krankheiten oder Parasiten (z. B. Everts 1995, Mallinger et al. 2017). Vor allem bei stark begrenztem Blütenangebot, wie es heute in der Agrarlandschaft der Fall ist, ist die Zunahme von Honigbienen aus Artenschutzsicht daher nicht erstrebenswert, da es doch vielmehr um die Förderung der Wildbienen und anderer Bestäuber gehen soll, auf die sich der drastische Rückgang bezieht. Im blütenreicheren urbanen Raum mit seinen zahlreichen exotischen Zierpflanzen kann die Imkerei jedoch gerade den naturfernen Stadtmenschen einen Zugang zum Thema bieten. So kann sich einiges, was weit-

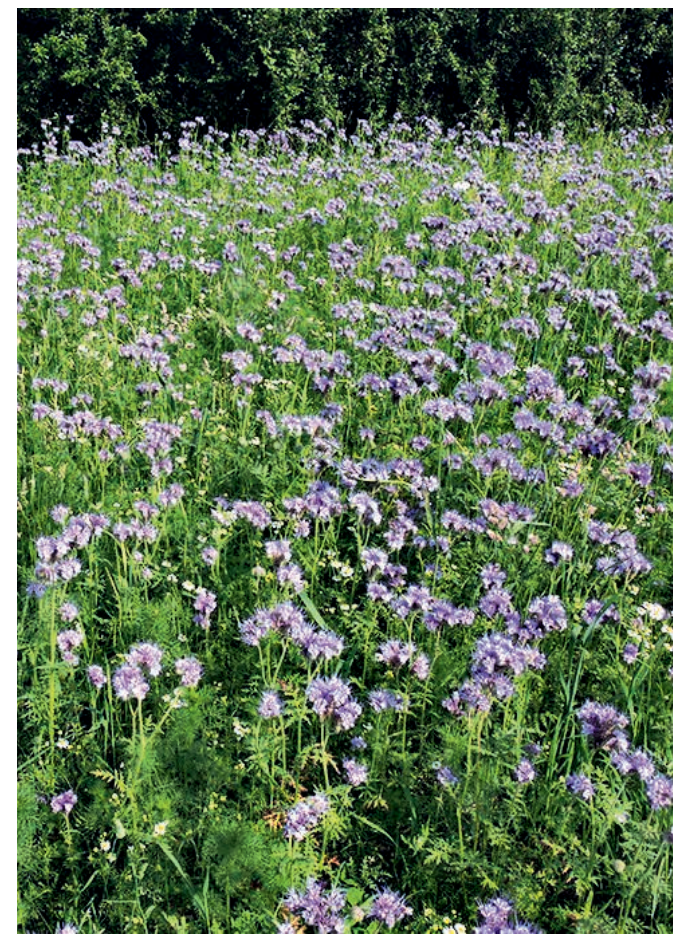

Abb. 11: Einjährige „Bienenweide“ mit Phazelie als Zwischensaat auf einem Acker, Geseke/Krs. Soest, 05.06.2016). (Foto: A. JAGEL)

gehend unreflektiert der Honigbiene helfen soll, auch positiv auf die Wildinsektenwelt auswirken, wenn man differenzierter agiert und nicht zur schnellen Lösung, der Samentüte vom Discounter, greift.

\section{Lösungsansätze}

Abgesehen von den vorherrschenden Verwirrungen und der Kommerzialisierung eines ernsten Problems, ist es zunächst erfreulich, dass überhaupt ein Naturschutzthema breite Aufmerksamkeit erlangt. Insbesondere aus dem Grund darf nun auf keinen Fall seitens des Naturschutzes das Gefühl vermittelt werden, dass spontane und eigenständige Aktionen grundsätzlich falsch oder „unprofessionell“ seien. Vielmehr sollte auf breiter Basis eine Anleitung stattfinden und weitere Aufklärung betrieben werden, zumal sich gerade dadurch die Chance bietet, neue, junge Mitstreiter für den Naturschutz zu gewinnen, den Nachwuchs zu fördern und Umweltbildung zu betreiben. 


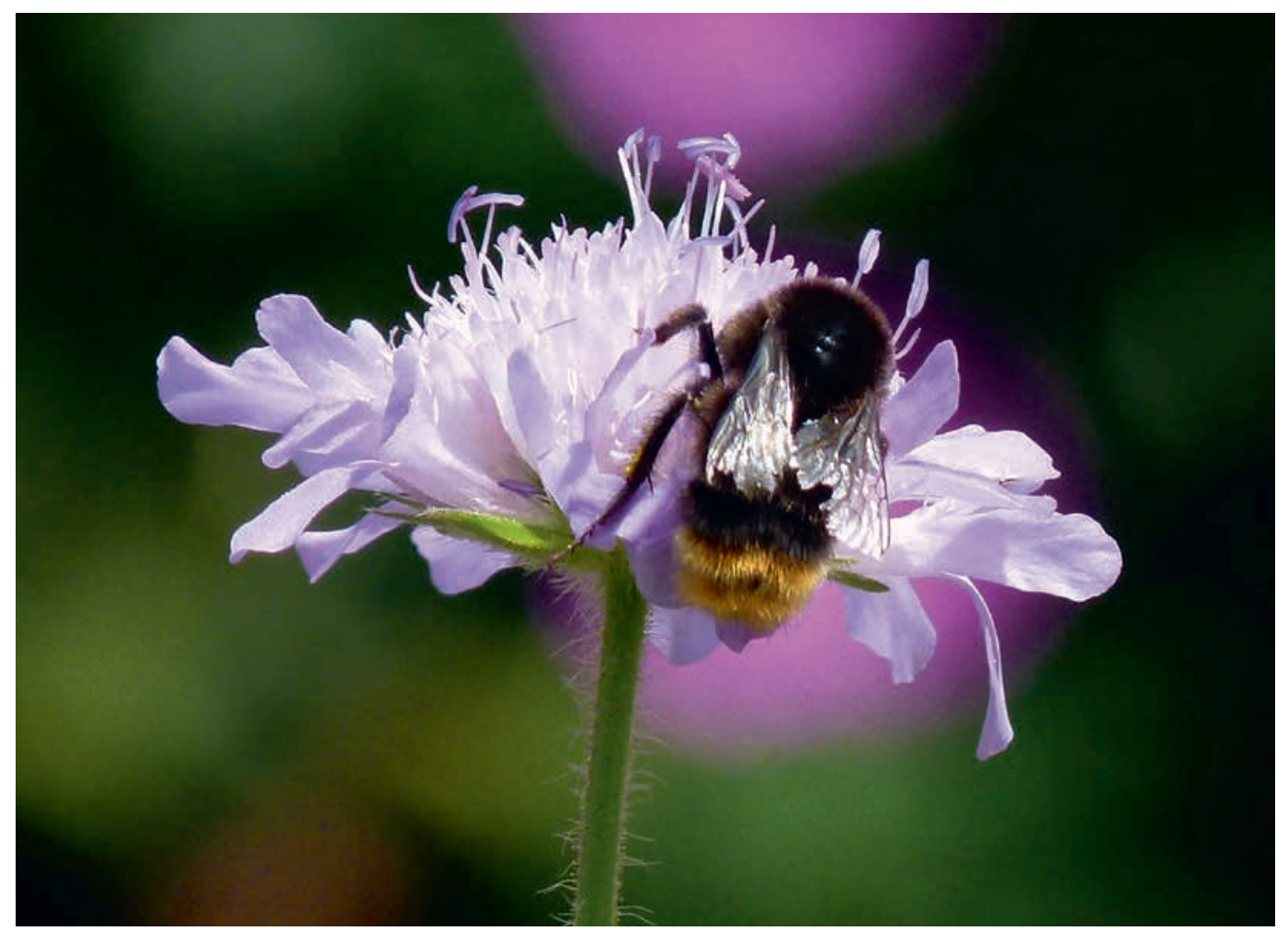

Abb. 12: Eine ideale Futterpflanze für Insekten ist die Acker-Witwenblume (Knautia arvensis). Die Staude kommt in mageren Wiesen vor. (Foto: H. STEINECKe)

Als Alternativen zur unbedachten Samenaussaat in der Landschaft wird hier eine Auswahl an Maßnahmen vorgeschlagen:

- Wiesen zunächst extensivieren: zweischürige Mahd von Glatthaferwiesen im Juni und Spätsommer, Abtransport und Verwertung des Mahdgutes, keine oder höchstens geringe Düngung. Bei ausbleibendem Erfolg hinsichtlich des anvisierten Blütenreichtums Anreicherung mit Regiosaatgut nach Anleitung, alternativ Mahdgutübertragung oder Anreicherung mit Heudrusch aus der Region.

- Bei einer Ansaat Arten wählen, die dem Naturraum und dem Standort angepasst sind; gegebenenfalls Literaturrecherche und eigene Kombinationen zusammenstellen, die von den Standardlisten der Regiosaatgutanbieter abweichen (vgl. BucH 2019). Einjährige Akzeptanzarten wie Klatsch-Mohn und Kornblume sind in der Wiese nicht nötig.
- Langfristige naturschutzkonforme Pflege solcher Flächen sichern, z. B. durch entsprechende Pachtverträge, Flächenerwerb, Ausgleichsflächen etc. Bei Obstwiesen: Grünland und Säume bei der Einrichtung, Entwicklung und Pflege stärker berücksichtigen.

- Säume und Randstreifen schaffen, Grünlandsäume einmal jährlich im Spätsommer mähen oder alternierend alle zwei Jahre, sodass ein Teil über den Winter stehen bleibt. Grünlandsäume nicht mulchen, sondern abräumen!

- Weder Pestizide noch Dünger anwenden

- Insektenschutz im eigenen Garten: Hier können manche Exoten (Zier- und Nutzpflanzen) durchaus nützlich sein. Tipps zu naturnahem Gärtnern berücksichtigen (Informationen bei Naturschutzverbänden oder Naturgartenvereinen). Regiosaatgut kann auch in kleinen Mengen und für den Privatgebrauch bestellt werden. 
- Geduld bewahren und bewusst machen, dass es bis zum Erfolg einige Jahre dauert, dieser aber nachhaltig ist.

\section{Fazit}

Der Schutz von Insekten, von Artenvielfalt im Allgemeinen und der Schutz von Natur und Landschaft ist ein kompliziertes Themenfeld. Die Probleme haben sich bereits seit vielen Jahrzehnten manifestiert und Ursache und Wirkung sind in ihrer Komplexität wissenschaftlich noch nicht hinlänglich untersucht. Selbst innerhalb der Fachwelt herrschen gegensätzliche Auffassungen und Missverständnisse vor, sodass es nur logisch ist, dass sich eine Lösung nicht einfach, schnell und billig aus der Tüte zaubern lässt.

Die Ansaat von Bienenweiden aus dem Baumarkt ist jedenfalls keine Lösung des Problems. Sie dient höchstens der Beruhigung des ökologischen Gewissens, bedient kommerzielle Interessen und lenkt von politisch unbequemen Entscheidungen ab, v. a. beim Umdenken im Bereich der industrialisierten Landwirtschaft und Massentierhaltung. Die wirklich gravierenden Probleme des Naturschutzes werden somit überdeckt und die Bevölkerung durch blinden Aktionismus beschäftigt. Zugespitzt formuliert wird eine grundfalsche Wahrnehmung von Natur und Artenschutz in die Öffentlichkeit transportiert.

Derzeit bietet sich nämlich eine einmalige Chance, die bereits vorhandene Aufmerksamkeit und Handlungsbereitschaft sinnvoll zu lenken und Projekte mit neuen Mitstreitern und neu entstehenden finanziellen Fördermöglichkeiten aufzutun. Während die Industrie und die Politik jedoch schnell aufgesattelt haben, müssen nun sowohl die Wissenschaft als auch der Naturschutz dringend öffentlich wirksam nachziehen und ihr Wissen, die langjährige Erfahrung und die bereits vorhandenen Ressourcen nutzen. Dies geht nur durch die $\mathrm{Zu}-$ sammenarbeit von Wissenschaft, professionellem und ehrenamtlichem Naturschutz, Behörden und aufgeschlossenen Vertretern der Landwirtschaft.

\section{Literatur}

BfN (Bundesamt für Naturschutz, Hrsg.) 2018: Rote Liste gefährdeter Tiere, Pflanzen und Pilze Deutschlands, Bd. 7: Pflanzen. - Naturschutz und Biologische Vielfalt 70(7).

Binot-Hafke, M., Balzer, S., Becker, N., Gruttke, H., Haupt, H., Hofbauer, N., Ludwig, G., Matzke-HaJek, G. \& Strauch, M. 2011: Rote Liste gefährdeter Tiere, Pflanzen und Pilze Deutschlands, Bd. 3: Wirbellose Tiere (Teil 1). - Bundesamt für Naturschutz, Bonn-Bad Godesberg.

Brosch, B., Hering, D., Jacobs, G., Keil, P., Korte, T. \& Loos, G. H. 2014: Urbane Biodiversität - ein Positionspapier. - Natur in NRW 2014(1): 41-44.

Buch, C. \& JAGEL, A. 2019: Schmetterlingswiese, Bienenschmaus und Hummelmagnet - Insektenrettung aus der Samentüte? - Veröff. Bochumer Bot. Ver. 11(2): 9-24.

Buch, C. \& KeIL, P. 2013: Industrienatur. Arbeitsmaterialien für Unterricht und Umweltbildung auf Industriebrachen im Ruhrgebiet. - Oberhausen.

Buch, C. 2018: Senecio inaequidens - Schmalblättriges Greiskraut (Asteraceae), Stadtpflanze des Jahres 2017. - Jahrb. Bochumer Bot. Ver. 9: 286-293.

Dehnen-Schmutz, K., Touza, J., Perrings, C. \& WilLIAMSON, M. 2007: The horticultural trade and ornamental plant invasions in Britain. - Conserv. Biol. 21: 224-231.

Dierschke, H. \& Briemle, G. 2002: Kulturgrasland. Wiesen, Weiden und verwandte Hochstaudengesellschaften. Stuttgart.

EverTs, S. 1995: Interspezifische Konkurrenz zwischen Honigbienen (Apis mellifera) und solitären Wildbienen (Hymenoptera, Apoidea). - Natur \& Landschaft 70: 165-172.

Floraweb 2019: http://www.floraweb.de/ [22.04.2019].

Hallmann, C. A., Sorg, M., Jongejans, E., Siepel, H., Hofland, N. \& Schwan, H. 2017: More than 75 percent decline over 27 years in total flying insect biomass in protected areas. - PLoS ONE 12(10): 1-21.

Jagel, A. \& Gausmann, P. 2010: Zum Wandel der Flora von Bochum im Ruhrgebiet (Nordrhein-Westfalen) in den letzten 120 Jahren. - Jahrb. Bochumer Bot. Ver. 1: 7-53.

Mallinger, R. E., Gaines-Day, H. R. \& Gratton, C. 2017: Do managed bees have negative effects on wild bees? A systematic review of the literature. - PloS ONE 12(12) https://journals.plos.org/plosone/article?id=10.1371/journal. pone.0189268 [05.05.2019].

Wittig, R. \& Niekisch, M. 2014: Biodiversität: Grundlagen, Gefährdung, Schutz. - Berlin, Heidelberg.

\section{Anschriften der Autorin und des Autors}

Corinne Buch, Klotzdelle 7a, 45472 Mülheim an der Ruhr, E-Mail: corinne.buch@botanik-bochum.de

Dr. Armin Jagel, Danziger Str. 2, 44789 Bochum, E-Mail: armin.jagel@botanik-bochum.de 